\title{
Theory of the friction force using electron cooling as an intrabeam scattering process
}

\author{
George Parzen \\ NOVEMBER 2006 \\ BNL REPORT C-A/AP NO.261
}

\begin{abstract}
Using the results found previously [5] for the cooling rates of the emittances, due to collisions between the electrons and the ions, a result is found for the friction force acting on the ions. It is shown that the friction force found here when used to track the ion bunch will give the same emittance cooling rates as those found using the intrabeam scattering theory for electron cooling [5].For the case of the uniform in space electron bunch distribution, the friction force found here agrees with the friction force result found with the usual theory of electron cooling.
\end{abstract}

\section{Introduction}

Using the results found previously [5] for the cooling rates of the emittances, due to collisions between the electrons and the ions, a result is found for the friction force acting on the ions. It is shown that the friction force found here when used to track the ion bunch will give the same emittance cooling rates as those found using the intrabeam scattering theory for electron cooling [5].For the case of the uniform in space electron bunch distribution, the friction force found here agrees with the friction force result found with the usual theory of electron cooling. 


\section{Intrabeam scattering, ions on ions}

Consider a beam which consists of a single bunch of completely ionized ions. The ions are doing betatron oscillations in the transverse direction, and synchrotron oscillations in the longitudinal direction. In addition the ions are subject to the coulomb repulsion between them. The scattering of each ion by the other ions is called intrabeam scattering. In Rhic, intrabeam scattering (IBS) causes the beam dimensions to grow slowly in all three diretions. The growth of the beam can be computed using intrabeam scattering theory [1-4].

\section{Intrabeam scattering, ions on electrons}

In electron cooling the ion bunch is overlapped by an electron bunch which is moving at the same velocity as the ion bunch. The ions can now scatter off each other or they can scatter off the electrons in the electron bunch. The scattering of the ions from each other occurs all around the accelerator ring and causes the emittances of the beam to grow. The scattering of the ions from the electrons occurs only in the cooling section and causes negative growth (cooling) in the ion emittances. Each kind of scattering may be considered as a kind of intrabeam scattering and the growth of the ion bunch due to each kind of scattering can be computed in the same way [5].

\section{Friction force definition using intrabeam scat- tering results for electron cooling}

The friction force will be defined as a force which acting on each ion in the ion bunch will produce the same cooling rates, due to collisions between ions and electrons, for the three quantities, $\left\langle p_{i}^{2}\right\rangle, \quad i=x, y, s$ for the ions as that found by the IBS theory for electron cooling. $\left\langle p_{i}^{2}\right\rangle$ is the average of $p_{i}^{2}$ over all the ions in the bunch. It will be shown below that the friction force found using this definition when used to track the ion bunch will give the same emittance cooling rates as those found using the IBS theory of electron cooling. 


\section{$5 \quad$ Friction force results found using intrabeam scattering results for electron cooling}

Using the results for the cooling rates of $\left\langle p_{i}^{2}\right\rangle, \quad i=x, y, s$ found by the IBS theory for electron cooling [5], and the above definition of the friction force, one finds the following expression for the friction force. The subscripts $a, b$ indicate ions and electrons respectively. $N_{b} f_{b}\left(x, v_{b}\right)$ is the electron distribution function. $N_{b}$ is the total nuber of electrons in the electron bunch. $x$ is the location of the ion. The derivation of the friction force results are given below. Using the Coulomb cross-section, one gets

$$
\begin{aligned}
& \sigma_{a b}=\left(\frac{r_{a b}}{\beta_{a b}^{2}}\right)^{2} \frac{1}{(1-\cos \theta)^{2}} \quad \text { coulomb cross }- \text { section in } C M S \\
& r_{a b}=\frac{Z_{a} Z_{b} e^{2}}{\mu c^{2}} \\
& \beta_{a b} c=\left|\overrightarrow{v_{a}}-\overrightarrow{v_{b}}\right| \\
& \frac{1}{\mu}=\frac{1}{m_{a}}+\frac{1}{m_{b}} \\
& F_{i}=-4 \pi m_{b} N_{b} r_{a b}^{2} c^{4} \int d^{3} v_{b} \frac{\left(v_{a}-v_{b}\right)_{i}}{\left|v_{a}-v_{b}\right|^{3}} f_{b}\left(x, v_{b}\right) \ln \left[\frac{\beta_{a b}^{2} b_{\operatorname{maxab}}}{r_{a b}}\right]
\end{aligned}
$$

One can also find a result for any $\sigma_{a b}$, and not just the coulomb $\sigma_{a b}$.

$$
F_{i}=-2 \pi m_{b} N_{b} \int d^{3} v_{b} \quad\left[\left(v_{a}-v_{b}\right)_{i}\left|v_{a}-v_{b}\right| f_{b}\left(x, v_{b}\right) \int d \theta \sin \theta(1-\cos \theta) \sigma_{a b}\right]
$$

\subsection{Uniform electron bunch case}

For a uniform in space electron beam

$$
\begin{aligned}
f_{b}\left(x, v_{b}\right) & =\frac{1}{\text { volume }} f_{v}\left(v_{b}\right) \\
n_{b} & =N_{b} / \text { volume electron density } \\
F_{i} & =-4 \pi m_{b} n_{b} r_{a b}^{2} c^{4} \int d^{3} v_{b} \frac{\left(v_{a}-v_{b}\right)_{i}}{\left|v_{a}-v_{b}\right|^{3}} f_{v}\left(v_{b}\right) \ln \left[\frac{\beta_{a b}^{2} b_{\text {maxab }}}{r_{a b}}\right]
\end{aligned}
$$


This result for the friction force for a uniform in space electron beam is the same as the result found using the usual theory of electron cooling.

\subsection{Gaussian bunch case}

$$
\begin{aligned}
f_{b}\left(x, v_{b}\right)= & \frac{\exp \left[-x^{2} /\left(2 \sigma_{x}^{2}\right)-y^{2} /\left(2 \sigma_{y}^{2}\right)-s^{2} /\left(2 \sigma_{s}^{2}\right)\right]}{(2 \pi)^{3 / 2} \sigma_{x} \sigma_{y} \sigma_{s}} f_{v}\left(v_{b}\right) \\
F_{i}= & -4 \pi m_{b} N_{b} r_{a b}^{2} c^{4} \frac{\exp \left[-x^{2} /\left(2 \sigma_{x}^{2}\right)-y^{2} /\left(2 \sigma_{y}^{2}\right)-s^{2} /\left(2 \sigma_{s}^{2}\right)\right]}{(2 \pi)^{3 / 2} \sigma_{x} \sigma_{y} \sigma_{s}} \\
& \int d^{3} v_{b} \frac{\left(v_{a}-v_{b}\right)_{i}}{\left|v_{a}-v_{b}\right|^{3}} f_{v}\left(v_{b}\right) \ln \left[\frac{\beta_{a b}^{2} b_{\text {maxab }}}{r_{a b}}\right]
\end{aligned}
$$

This result can be generalized to apply to any electron bunch ditribution that can be factored and written as

$$
f_{b}\left(x, v_{b}\right)=f_{x}(x) f_{v}\left(v_{b}\right)
$$

One then finds

$$
F_{i}=-4 \pi m_{b} N_{b} r_{a b}^{2} c^{4} f_{x}(x) \int d^{3} v_{b} \frac{\left(v_{a}-v_{b}\right)_{i}}{\left|v_{a}-v_{b}\right|^{3}} f_{v}\left(v_{b}\right) \ln \left[\frac{\beta_{a b}^{2} b_{\text {maxab }}}{r_{a b}}\right]
$$

The results for the friction force given in this paper may differ from the usual friction force results when the electron bunch distribution can not be factored. This may happen when the alpha funtion is not zero or when dispersion is present.

\section{Cooling rates for $<p_{i} p_{j}>$, due to collisions, and for $<x_{i} p_{i}>$}

If a horizontal dispersion is present in the cooling section, then the cooling rate of the emittances will also depend on the cooling rate of $<p_{x} p_{s}>$. It will be shown that the friction force obtained as described above when used to track a particle sample of the ion diStribution will give the same cooling rate 
for $<p_{x} p_{s}>$ as that found using the IBS theory of electron cooling. Similar statements can be made for the vertical dispersion. Thus the friction force can be used to track a bunch of ions when dispersion is present to find the same emittance cooling rates as those found using the IBS theory of electron cooling.

The friction force as defined here to give the correct cooling rates, due to collisions, for $\left\langle p_{i}^{2}\right\rangle, \quad i=x, y, s$ also gives the correct cooling rates for all 6 of the moments $\left\langle p_{i} p_{j}\right\rangle, \quad i, j=x, y, s$. It will also be shown that it gives the correct cooling rates, due to collisions, for $\left\langle x_{i} p_{i}\right\rangle, \quad i=x, y, s$ which is required to compute the cooling rates of the emittances.

\section{Derivation of the friction force using intra- beam scattering results for electron cooling}

To derive the results for the friction force, we will first find the cooling rates for $\left\langle p_{i}^{2}>, \quad i=x, y, s\right.$, due to collisions, using the friction force. We will then find the cooling rates for $\left\langle p_{i}^{2}\right\rangle, \quad i=x, y, s$ using the methods of IBS. Comparing these two results for the cooling rates, due to collisions, for $<p_{i}^{2}>, \quad i=x, y, s$ will give us the result for the friction force.

\subsection{Cooling rate of $\left\langle p_{i}^{2}>\right.$ from the friction force}

Let $p_{i k}, \quad i=x, y, s$ be the components of the momentum of the $k$ th ion. Let $v_{i k}, \quad i=x, y, s$ be the components of the ion velocity. Let $N_{a}$ be the number of ions in the ion bunch. Let $F_{i}$ be the components of the friction force acting on the ion. If the ions are tracked using this friction force then

$$
\begin{aligned}
\frac{d p_{i k}}{d t} & =F_{i} \\
\frac{d p_{i k}^{2}}{d t} & =2 m_{a} v_{i k} F_{i} \\
\frac{d<p_{i k}^{2}>}{d t} & =\frac{1}{N_{a}} \sum_{k=1}^{N_{a}} 2 m_{a} v_{i k} F_{i} \\
\frac{d<p_{i a}^{2}>}{d t} & =\int d^{3} x d^{3} v_{a} f_{a}\left(x, v_{a}\right) 2 m_{a} v_{i a} F_{i}
\end{aligned}
$$


Note that $d / d t$ here gives only the rate of change of the relevant quantity. due to collisions between ions and electrons.

\subsection{Cooling rate of $<p_{i}^{2}>$ from the IBS theory of elec- tron cooling}

Let $\delta N_{a}$ be the number of ions with momentum, $p_{a}$ in $d^{3} p_{a}$ and space coordinate $x$ in $d^{3} x$ which are scattered by the electrons with momentum $p_{b}$ in $d^{3} p_{b}$ which are also in $d^{3} x$, in the time interval $d t$, into the solid angle $d \Omega$. In a scattering event $p_{a}, p_{b}$ change to $p_{a}^{\prime}, p_{b}^{\prime}$ and $q_{a}=p_{a}^{\prime}-p_{a}$ is the change in the ion momentum. Then $\delta N_{a}$ is given by, Ref.[4,5],

$$
\delta N_{a}=d \Omega \quad \sigma_{a b} \quad N_{a} f_{a}\left(x, v_{a}\right) d^{3} v_{a}\left|v_{a}-v_{b}\right| \quad N_{b} f_{b}\left(x, v_{b}\right) d^{3} v_{b} d^{3} x \quad d t
$$

$\sigma_{a b}$ is the scattering cross section for the scattering of the ions from the electrons. Using this result for $\delta N_{a}$ one can find that $[4,5]$

$$
\begin{aligned}
\delta<p_{i a}^{2}>= & \int d^{3} v_{b} d^{3} x d^{3} v_{a}\left[f_{a}\left(x, v_{a}\right) N_{b} f_{b}\left(x, v_{b}\right)\left|v_{a}-v_{b}\right|\right. \\
& \left.\int d \Omega \sigma_{a b} \delta\left(p_{i a}^{2}\right)\right] d t \\
\delta\left(p_{i a}^{2}\right)= & \left(p_{i a}+q_{i a}\right)^{2}-p_{i a}^{2} \\
= & 2 p_{i a} q_{i a}+q_{i a}^{2} \\
= & 2 p_{i a} q_{i a} \quad d r o p p i n g q_{i a}^{2} \text { (see below) } \\
\int d \Omega \quad \sigma_{a b} \delta\left(p_{i a}^{2}\right)= & \int d \Omega \quad \sigma_{a b} 2 p_{i a} q_{i a} \\
q_{i a}= & p_{i a}^{\prime}-p_{i a}
\end{aligned}
$$

In Eq.6, $p_{i a}$ does not depend on the scattering angles $\theta, \phi$. Let $d_{i}$ be defined as

$$
d_{i}=\int d \Omega \quad \sigma_{a b} q_{i a}
$$


$d \Omega \sigma_{a b}$ is an invariant and $q_{i a}$ is a vector in 3-space which has the same magnitude in the Rest CS and in the Center of Mass CS (CMS). Then $d_{i}$ is a vector in 3-space and can be evaluated in the CMS.

If this integral is evaluated in the CMS and the result is written in terms of tensors in 3-space then the result will also hold in the Rest CS.

In the CMS, we introduce a polar coordinate system $\theta, \phi$ where $\theta$ is measured relative to the direction of $\overrightarrow{p_{a}}$ and we assume that $\sigma_{a b}(\theta, \phi)$ is a fumction of $\theta$ only. we can then write

$$
\begin{aligned}
\overrightarrow{p_{a}} & =(0,0,1)\left|\overrightarrow{p_{a}}\right| \\
\overrightarrow{p_{a}^{\prime}} & =(\sin \theta \cos \phi, \sin \theta \sin \phi, \cos \theta)\left|\overrightarrow{p_{a}}\right| \\
\overrightarrow{q_{a}} & =(\sin \theta \cos \phi, \sin \theta \sin \phi, \cos \theta-1)\left|\overrightarrow{p_{a}}\right|
\end{aligned}
$$

In the CMS, using Eq.7, one finds

$$
d_{i}=-2 \pi \int d \theta \sin \theta(1-\cos \theta) \sigma_{a b}(0,0,1)\left|p_{a}\right|
$$

These results for $d_{i}$ in the CMS can be rewritten in terms of tensors in 3space. In the CMS

$$
\begin{gathered}
v_{i a}-v_{i b}=p_{i a} / m_{a}-p_{i b} / m_{b}=p_{i a} / \mu \\
p_{i a}=\mu\left(v_{i a}-v_{i b}\right)
\end{gathered}
$$

and

$$
d_{i}=-2 \pi \int d \theta \sin \theta(1-\cos \theta) \sigma_{a b} \mu\left(v_{i a}-v_{i b}\right)
$$

In this form the result will also hold in the Rest CS.

Using the above results for $\delta\left(p_{i a}^{2}\right)$, due to collisions, and for $d_{i}$ and putting them into the result for $\delta<p_{i a}^{2}>$ in Eq.6, one finds 


$$
\begin{aligned}
\delta<p_{i a}^{2}>= & \int d^{3} x d^{3} v_{a} f_{a}\left(x, v_{a}\right) 2 m_{a} v_{i a} \\
& {\left[-2 \pi m_{b} \int d^{3} v_{b} N_{b}\left(v_{a}-v_{b}\right)_{i}\left|v_{a}-v_{b}\right| f_{b}\left(x, v_{b}\right)\right.} \\
& \left.\left(\int d \theta \sin \theta(1-\cos \theta) \sigma_{a b}\right) \quad d t\right]
\end{aligned}
$$

\subsection{Friction force results}

Comparing the result for $\delta<p_{i a}^{2}>$, due to collisions, found here with the result for $\delta<p_{i a}^{2}>$ found in section 7.1, we get the result for the friction force

$$
F_{i}=-2 \pi m_{b} N_{b} \int d^{3} v_{b} \quad\left[\left(v_{a}-v_{b}\right)_{i}\left|v_{a}-v_{b}\right| f_{b}\left(x, v_{b}\right) \int d \theta \sin \theta(1-\cos \theta) \sigma_{a b}\right]
$$

Using for $s i g_{a b}$ the results for the coulomb croos-section given in Eq.1 one finds

$$
\begin{aligned}
\sigma_{a b} & =\left(\frac{r_{a b}}{\beta_{a b}^{2}}\right)^{2} \frac{1}{(1-\cos \theta)^{2}} \quad \text { coulomb cross - section in CMS } \\
r_{a b} & =\frac{Z_{a} Z_{b} e^{2}}{\mu c^{2}} \\
\beta_{a b} c & =\left|\overrightarrow{v_{a}}-\overrightarrow{v_{b}}\right| \\
\frac{1}{\mu} & =\frac{1}{m_{a}}+\frac{1}{m_{b}} \\
F_{i} & =-4 \pi m_{b} N_{b} r_{a b}^{2} c^{4} \int d^{3} v_{b} \frac{\left(v_{a}-v_{b}\right)_{i}}{\left|v_{a}-v_{b}\right|^{3}} f_{b}\left(x, v_{b}\right) \ln \left[\frac{\beta_{a b}^{2} b_{\text {maxab }}}{r_{a b}}\right]
\end{aligned}
$$

We can now justify dropping the $q_{i a}^{2}$ term in Eq.6. We will show that $\left|q_{a}\right|$ is smaller than $\left|p_{a}\right|$ in the Rest CS by the factor $m_{b} / m_{a}$. Thus the $q_{i a}^{2}$ term in Eq. 6 is smaller than the $2 p_{i a} q_{i a}$ by the factor $m_{b} / m_{a}$.

$\left|q_{a}\right|$ has the same vaue in the CMS and in the Rest CS. In the CMS $\left|q_{a}\right|$ has the magnitude of $\left|p_{a}\right|$ in the CMS. In Rhic, $\left|q_{a}\right|$ has the magnitude of $1 e-3 m_{b} c$ while $\left|p_{a}\right|$ in the Rest CS has the magnitude of $1 e-3 m_{a} c$. Thus $\left|q_{a}\right|$ is smaller than $\left|p_{a}\right|$ in the Rest CS by the factor $m_{b} / m_{a}$. 


\subsection{Cooling rates for $\left\langle p_{i} p_{j}>\right.$, due to collisions, re- quired when dispersion is present}

If a horizontal dispersion is present in the cooling section, then the cooling rate of the emittances will also depend on the cooling rate of $\left\langle p_{x} p_{s}\right\rangle$, due to collisions. It will be shown that the friction force obtained as described above when used to track a particle sample of the ion ditribution will give the same cooling rate, due to collisions, for $\left\langle p_{x} p_{s}\right\rangle$ as that found using the IBS theory of electron cooling. Similar statements can be made for the vertical dispersion. Thus the friction force can be used to track a bunch of ions when dispersion is present to find the same emittance cooling rates as those found using the IBS theory of electron cooling.

First let us find the cooling rate of $\left\langle p_{i a} p_{j a}>\right.$ using the friction force. Using the same procedure as given in section 7.1 one gets

$$
\begin{aligned}
\frac{d p_{i k}}{d t} & =F_{i} \\
\frac{d\left(p_{i k} p_{j k}\right)}{d t} & =m_{a}\left(v_{i k} F_{j}+v_{j k} F_{i}\right) \\
\frac{d<p_{i k} p_{j k}>}{d t} & =\frac{1}{N_{a}} \sum_{k=1}^{N_{a}} m_{a}\left(v_{i k} F_{j}+v_{j k} F_{i}\right) \\
\frac{d<p_{i a} p_{j a}>}{d t} & =\int d^{3} x d^{3} v_{a} f_{a}\left(x, v_{a}\right) m_{a}\left(v_{i a} F_{j}+v_{j a} F_{i}\right)
\end{aligned}
$$

This result for the cooling rate of $\left\langle p_{i a} p_{j a}\right\rangle$, due to collisions, found using our result for the friction force will now be shown to be the same result as that found using the IBS theory of electron cooling [5]. The cooling rate

of $<p_{i a} p_{j a}>$ using the IBS theory of electron cooling can be found using the the same procedure as that given in section 7.2 .

$$
\begin{aligned}
\delta<p_{i a} p_{j a}>= & \int d^{3} v_{b} d^{3} x d^{3} v_{a}\left[f_{a}\left(x, v_{a}\right) N_{b} f_{b}\left(x, v_{b}\right)\left|v_{a}-v_{b}\right|\right. \\
& \left.\int d \Omega \sigma_{a b} \delta\left(p_{i a} p_{j a}\right)\right] \\
\delta\left(p_{i a} p_{j a}\right)= & \left(p_{i a}+q_{i a}\right)\left(p_{j a}+q_{j a}\right)-p_{i a} p_{j a} \\
= & p_{i a} q_{j a}+p_{j a} q_{i a}+q_{i a} q_{j a}
\end{aligned}
$$




$$
\begin{aligned}
& =p_{i a} q_{j a}+p_{j a} q_{i a} \quad \text { dropping } q_{i a} q_{j a} \\
\int d \Omega \quad \sigma_{a b} \delta\left(p_{i a} p_{j a}\right) & =\int d \Omega \quad \sigma_{a b}\left(p_{i a} q_{j a}+p_{j a} q_{i a}\right) \\
q_{a} & =p_{a}^{\prime}-p_{a}
\end{aligned}
$$

Eq.14 now becomes

$$
\begin{aligned}
\delta<p_{i a} p_{j a}>= & \int d^{3} x d^{3} v_{a} f_{a}\left(x, v_{a}\right) m_{a} \\
& \left(-2 \pi m_{b} \int d^{3} v_{b}\left(v_{i a}\left(v_{a}-v_{b}\right)_{j}+v_{j} a\left(v_{a}-v_{b}\right)_{i}\right)\left|v_{a}-v_{b}\right|\right. \\
& \left.N_{b} f_{b}\left(x, v_{b}\right) \quad\left(\int d \theta \sin \theta(1-\cos \theta) \sigma_{a b}\right) \quad d t\right)
\end{aligned}
$$

which, using Eq.11 for the friction force, can be written as

$$
\delta<p_{i a} p_{j a}>=\int d^{3} x d^{3} v_{a} f_{a}\left(x, v_{a}\right) m_{a}\left(v_{i a} F_{j}+v_{j a} F_{i}\right) d t
$$

This is the same result as that found using the friction force, Eq.13.

\subsection{Cooling rates for $\left\langle x_{i} p_{i}\right\rangle$, due to collisions.}

First let us find the cooling rate of $\left\langle x_{i} p_{i a}\right\rangle$ using the friction force. Using the same procedure as given in section 7.1 one gets

$$
\begin{aligned}
\frac{d p_{i k}}{d t} & =F_{i} \\
\frac{d\left(x_{i k} p_{i k}\right)}{d t} & =x_{i k} F_{i} \\
\frac{d<x_{i k} p_{i k}>}{d t} & =\frac{1}{N_{a}} \sum_{k=1}^{N_{a}} x_{i k} F_{i} \\
\frac{d<x_{i} p_{i a}>}{d t} & =\int d^{3} x d^{3} v_{a} f_{a}\left(x, v_{a}\right) x_{i} F_{i}
\end{aligned}
$$


Note we are finding only the cooling rate due to collisions and in collisions $x$ does not change.

This result for cooling rate of $\left\langle x_{i} p_{i a}\right\rangle$, due to collisions, found using our result for the friction force will now be shown to be the same result as that found using the IBS theory of electron cooling [5]. The cooling rate of $\left\langle x_{i} p_{i a}>\right.$ using the IBS theory of electron cooling can be found using the the same procedure as that given in section 7.2 .

$$
\begin{aligned}
\delta<x_{i} p_{i a}>= & \int d^{3} v_{b} d^{3} x d^{3} v_{a}\left[f_{a}\left(x, v_{a}\right) N_{b} f_{b}\left(x, v_{b}\right)\left|v_{a}-v_{b}\right|\right. \\
& \left.\int d \Omega \sigma_{a b} \delta\left(x_{i} p_{i a}\right)\right] \\
\delta\left(x_{i} p_{i a}\right)= & x_{i} q_{i a} \\
\int d \Omega \quad \sigma_{a b} \delta\left(p_{i a} p_{j a}\right)= & \int d \Omega \quad \sigma_{a b} x_{i} q_{i a} \\
q_{i a}= & p_{i a}^{\prime}-p_{i a}
\end{aligned}
$$

Eq.18 now becomes, using EQ.9 FOR $\int d \Omega \sigma_{a b} q_{i a}$

$$
\begin{aligned}
\delta<x_{i} p_{i a}>= & \int d^{3} x d^{3} v_{a} f_{a}\left(x, v_{a}\right) \\
& \left(-2 \pi m_{b} \int d^{3} v_{b} x_{i}\left(v_{a}-v_{b}\right)_{i}\left|v_{a}-v_{b}\right|\right. \\
& \left.N_{b} f_{b}\left(x, v_{b}\right) \quad\left(\int d \theta \sin \theta(1-\cos \theta) \sigma_{a b}\right) \quad d t\right)
\end{aligned}
$$

which, using Eq.11 for the friction force, can be written as

$$
\delta<x_{i} p_{i a}>=\int d^{3} x d^{3} v_{a} f_{a}\left(x, v_{a}\right) x_{i} F_{i} d t
$$

This is the same result as that found using the friction force, Eq.17.

Thanks are due to Alexei Fedotov for his assistance in comparing the results of the IBS treatment of electron cooling and the results found using the friction force. 


\section{References}

1. A. Piwinski Proc. 9th Int. Conf. on High Energy Accelerators (1974) 405

2. J.D. Bjorken and S.K. Mtingwa, Part. Accel.13 (1983) 115

3. M. Martini CERN PS/84-9 (1984)

4. G. Parzen BNL report C-A/AP/N0.150 (2004) and at http://arxiv.org/ps_ cache/physics/pdf/0405/0405019.pdf

5. G. Parzen BNL report C-A/AP/N0.243 (2006) and at http://arxiv.org/abs/physics/0609076 